\title{
The INSPIRE Comparative Cost Study: I2-Month Health Economic and Clinical Outcomes Associated with Hysterectomy, Myomectomy, and Treatment with the Sonata System
}

This article was published in the following Dove Press journal:

ClinicoEconomics and Outcomes Research

\author{
Elizabeth Brooks (D) \\ Linda Mihalov $\mathbb{D}^{2}$ \\ Dipak Delvadia $\mathbb{1}^{3}$ \\ Joseph Hudgens ${ }^{4}$ \\ Saifuddin Mama $\left.{ }^{5}\right)^{5}$ \\ Gretchen E Makai ${ }^{6}$ \\ Matt W Yuen' \\ Carter A Little' \\ Robert L Bauserman' \\ April Zambelli-Weiner (D) \\ David J Levine ${ }^{7}$ \\ 'TTi Health Research \& Economics, \\ Westminster, MD, USA; ${ }^{2}$ Benaroya \\ Research Institute at Virginia Mason \\ Medical Center, Seattle, WA, USA; \\ ${ }^{3}$ Drexel University College of Medicine, \\ Philadelphia, PA, USA; ${ }^{4}$ Eastern Virginia \\ Medical School, Norfolk, VA, USA; \\ ${ }^{5}$ Cooper Medical School of Rowan \\ University, Camden, NJ, USA; ${ }^{6}$ Christiana \\ Care Health System, Newark, DE, USA; \\ ${ }^{7}$ Mercy Clinical Minimally Invasive \\ Gynecology, St. Louis, MO, USA
}

Correspondence: Elizabeth Brooks

TTi Health Research \& Economics, I23 I

Tech Court, Suite 20I, Westminster, MD

21157, USA

Tel $+1800-580-2990$

Fax $+1888-800-580-2990$

Email ebrooks@tti-research.com
Purpose: The INSPIRE study compared perioperative and 12-month health economic and clinical outcomes associated with hysterectomy, myomectomy, and sonography-guided transcervical fibroid ablation (TFA) using the Sonata ${ }^{\circledR}$ system.

Patients and Methods: Cost and health care resource utilization (HCRU) data for TFA were obtained from a prospective, multicenter, single-arm clinical trial. Data for hysterectomy and myomectomy arms were derived from the Truven Health MarketScan commercial payer claims database. The Truven data was used to determine health economic outcomes and costs for the hysterectomy and myomectomy arms. For each arm, payer perspective costs were estimated from the available charge and HCRU data.

Results: TFA with Sonata had significantly lower mean length of stay (LOS) of $5 \mathrm{hrs}$ versus hysterectomy (73 hrs) or myomectomy ( $79 \mathrm{hrs;}$ all $p<0.001)$. The average payer cost for TFA treatment, including the associated postoperative HCRU was $\$ 8,941$. This was significantly lower compared to hysterectomy $(\$ 24,156)$ and myomectomy $(\$ 22,784$; all $p<$ $0.001)$. In the TFA arm, there were no device- or procedure-related costs associated with complications during the peri- or postoperative time frame. TFA subjects had significantly lower costs associated with complications, prescription medications, and radiology.

Conclusion: Compared to hysterectomy and myomectomy, TFA treatment with the Sonata system was associated with significantly lower index procedure cost, complication cost, and LOS, contributing to a lower total payer cost through 12 months.

Keywords: uterine fibroids, health care resource utilization, payer perspective analysis, transcervical fibroid ablation, TFA, uterine preserving treatment

\section{Introduction}

Uterine fibroids, also known as leiomyomata uteri, are benign tumors of the uterus that frequently occur in women of reproductive age. Uterine fibroids may be associated with heavy menstrual bleeding, dysmenorrhea, pelvic pain, decreased quality of life, and subfertility. ${ }^{1}$ Uterine fibroids are highly prevalent, with approximately $70 \%$ to $80 \%$ of premenopausal women likely to develop uterine fibroids prior to menopause. ${ }^{1,2}$ Among premenopausal women aged 40-49 years with uterine fibroids, $25 \%$ to $50 \%$ will develop clinical symptoms. ${ }^{2}$ Causal factors are not fully understood. However, certain risk factors are known, including epigenetic factors, concentrations of steroid hormones and growth factors, obesity, and being of Afro-Caribbean descent. ${ }^{3}$ 
Hysterectomy and myomectomy are the most commonly performed surgical interventions for the treatment of uterine fibroids. ${ }^{4}$ Hysterectomy involves extirpation of the uterus (and generally, the cervix), with or without ovarian conservation. Myomectomy is an operation in which individual fibroids are removed, retaining the uterus and the potential for pregnancy. According to a report from the Healthcare Cost and Utilization Project, myomectomies represented about $22 \%$ of operations for uterine fibroid treatment in the US during 2013 in both inpatient and ambulatory settings, while hysterectomies constituted more than $75 \%$ of the inpatient surgeries and $67 \%$ of the hospital-based ambulatory operations. ${ }^{4-6}$ One costeffectiveness study estimated that major complications within 30 days of surgery averaged $\$ 2,094$ for either hysterectomy or myomectomy. ${ }^{7}$ Recent studies estimated average payer costs for hysterectomy ranging from $\$ 16,184-\$ 25,499$ (2016 dollars), depending upon the procedure setting and route of surgery. ${ }^{8,9}$ A 2014 comparative effectiveness study of uterine fibroid treatments estimated the combined payer and patient direct cost per myomectomy to be $\$ 23,983 .{ }^{10}$ With over two hundred thousand combined procedures performed per year, this would equate to nearly five hundred million dollars in complication costs per year for hysterectomies and myomectomies in the United States alone. ${ }^{11}$

It is useful to understand both the clinical outcomes and the related payer costs associated with emerging treatment options compared to current standards of care such as hysterectomy and myomectomy. The Sonata ${ }^{\circledR}$ system provides a transcervical fibroid treatment (TFA) for symptomatic uterine fibroids. It is a sonography-guided, incisionless, and uterus-preserving radiofrequency ablation treatment and is approved and commercially available in Europe and the United States. Sonata utilizes a single-use radiofrequency ablation handpiece connected to a reusable intrauterine ultrasound probe, forming a single integrated treatment device designed to ablate uterine fibroids. This integration of realtime ultrasound (for imaging) with radiofrequency ablation (for treatment) in a single device, enables a gynecologist to visualize, target and ablate a greater range of fibroid types transcervically than can be approached via operative hysteroscopy. Operative hysteroscopy is limited to the treatment of intracavitary and smaller submucosal fibroids; at least in the case of submucosal fibroid, it represents a gold standard for treatment. ${ }^{12}$ However, Sonata uses intrauterine ultrasound to image and treat all non-pedunculated fibroids.
To better understand health care resource utilization (HCRU), procedure outcomes, and associated costs, we undertook a combined prospective and retrospective cohort study comparing TFA with Sonata to hysterectomy and myomectomy.

\section{Materials and Methods}

For the TFA procedure (Sonata ${ }^{\circledR}$ System, Gynesonics, Inc. Redwood City, CA, USA), HCRU, along with procedural and 12-month postoperative charges, were obtained from a subset of sites participating in the SONATA pivotal Investigational Device Exemption (IDE) clinical trial. SONATA was a prospective, longitudinal, multicenter, single-arm cohort clinical trial to assess the safety and efficacy of the Sonata system in the treatment of symptomatic uterine fibroids and enrolled patients between April 2015 and October 2016. ${ }^{13}$ Procedural and 12-month postoperative cost and outcomes data for the hysterectomy and myomectomy arms were obtained from a nationally representative administrative claims database (the Truven Health MarketScan commercial payer claims database) for the period from July 1, 2012 to June 30, 2014 as it was the most recent data available from Truven at the time of the data request. There were no specific sample size calculations due to the need to retrospectively obtain charge data from sites that had already participated in the prospective SONATA trial. It was anticipated that only some of those sites would be able to participate and that sample size for the Sonata group would be limited in this way.

\section{Study Population}

TFA arm included patients who were enrolled in the prospective clinical trial of the Sonata procedure (SONATA pivotal IDE trial) and consisted of premenopausal women 25-50 years of age, with heavy menstrual bleeding (HMB) associated with up to 10 fibroids from $1-5 \mathrm{~cm}$ in maximal diameter. $^{13}$

The hysterectomy and myomectomy patients included in the claims database analysis were women 25-50 years of age who reported one of the following: a claim with a uterine fibroid diagnosis on the same day as the index procedure; an inpatient claim with a uterine fibroid diagnosis at any time in the 12-month pre-index period; or at least two outpatient claims, occurring 30 days apart or more, with a uterine fibroid diagnosis at any time in the 12-month preindex period. Hysterectomy and myomectomy procedures of interest were identified using appropriate International Classification of Diseases, Ninth Revision, Clinical 
Modification (ICD-9-CM), International Classification of Disease, Tenth Revision, and Current Procedural Terminology (CPT) codes describing the procedures (Table 1). Patients included met the additional criteria of having had no uterine fibroid treatment procedure in the 42 month pre-index period; no diagnosis associated with postmenopause in either the 42-month pre-index period or the 24-month post-index period; no diagnosis for an extrauterine pelvic mass or abdominal/pelvic malignancy in the 24-month post-index period; and 42 months of pre-index and 24 months of post-index continuous medical and pharmacy enrollment.

The Western IRB (WIRB) determined that this research was exempt from IRB approval on $9 / 11 / 2017$, finding that it did not involve human subjects under 45 CFR 46.102(f). The finding was based on both the research not involving data collected specifically for the current project and the researchers not being able to ascertain the identity of individual participants.

\section{Outcomes}

The primary outcomes of interest for the Initial Comparison of Payer Costs for Sonata Relative to Standard of Care (INSPIRE) study were payer costs associated with the index

Table I Codes Used for Procedure Identification

\begin{tabular}{|c|c|c|}
\hline Procedure & ICD-9 & CPT \\
\hline \multicolumn{3}{|l|}{ Hysterectomy } \\
\hline Hysterectomy - abdominal & $68.39,68.49$ & $58150,58152,58180$ \\
\hline Hysterectomy - vaginal & 68.59 & $\begin{array}{l}58260,58262,58263, \\
58267,58270,58275, \\
58280,58290,58291, \\
58292,58293,58294\end{array}$ \\
\hline Hysterectomy - laparoscopic & $68.31,68.41$ & $\begin{array}{l}5854 I, 58542,58543, \\
58544,58570,5857 I, \\
58572,58573\end{array}$ \\
\hline $\begin{array}{l}\text { Hysterectomy - } \\
\text { laparoscopic vaginal }\end{array}$ & 68.51 & $\begin{array}{l}58550,58552,58553, \\
58554\end{array}$ \\
\hline \multicolumn{3}{|l|}{ Myomectomy } \\
\hline Myomectomy & 68.29 & $58|40,58| 45,58 \mid 46$ \\
\hline Myomectomy - laproscopic & & 58545,58546 \\
\hline Myomectomy - hysterscopic & & 58561 \\
\hline \multicolumn{3}{|c|}{ Codes used if robotic assistance was used } \\
\hline Robotic assistance & $\begin{array}{l}17.41,17.42 \\
17.49\end{array}$ & S2900 \\
\hline
\end{tabular}

procedure and the postoperative HCRUs through 12-months. The perioperative period was defined as the hospital stay for the index procedure. The postoperative period was defined as the period after discharge through 1 year. Postoperative HCRU categories included complication related HCRU, medications including opioid usage, radiology, office visits, supply costs, procedure-related complications, and subsequent procedures.

As part of the SONATA trial protocol, participating sites submitted institution billing form (UB-04) and/or provider billing form (HCFA-1500) for all charges accrued during the enrolled patients' Sonata procedure. The associated hospital outpatient payment is estimated based on Medicare 2016 Outpatient Prospective Payment System rates that would be associated with the Sonata procedure code 0404T $(\$ 5,699)$, which was effective January 1, 2016; all hospital outpatient services provided on the day of the procedure were bundled into one payment, as the code is a $\mathrm{J} 1$ status indicator. The physician payment for the TFA was based on average 2017 Medicare rates, which includes surgeon and anesthesiologist, and payments. We estimated for this study the surgeon payment (CPT code 0404T) would be comparable to that of laparoscopic radiofrequency ablation (CPT code 58674) and assigned the corresponding Medicare 2017 national payment. The anesthesiologist payment (CPT code 00852) was based on the 2016 Medicare base anesthesia unit payment (4.0). Five (5) time units (15 min increments per time unit), or 75 mins, were used to estimate the average anesthesia duration. This estimate is a conservative assessment based on the average operating room time of 77 mins for the TFA patients. This was then multiplied by the anesthesia 2016 conversion factor of $\$ 22.4426$, to arrive at the total estimated payment for the anesthesiologist services. Office visits were assumed to be coded as established patients, and CPT code 99213 was used. As the Sonata procedure reimbursement is carrier dependent, payments for all hospital and physician fees were then adjusted by a Medicare-to-commercial payer ratio of $125 \%{ }^{14}$ Prescription costs were based on RED BOOK Online (Truven Health Analytics, accessed December 2017). ${ }^{15}$

For the hysterectomy and myomectomy arms, patientlevel charge data associated with the index procedure and 12month postoperative HCRU data from the Truven dataset were converted to payments using a $70 \%$ charge-to-payment adjustment. ${ }^{16}$ The robustness of the result was assessed by varying this parameter in several sensitivity analyses. For each procedure, the following were collected: inpatient and outpatient facility charges, professional service charges, complication-related charges, postoperative outpatient office visits, prescription medications including opioids, ED visits, 
inpatient hospitalizations, length of stay, subsequent procedures, and radiology.

Several sensitivity analyses were conducted around the procedural and 12-month postoperative HCRU payment estimates. The Medicare-to-commercial payer adjustment used to estimate payments for TFA was increased to as much as $188 \%$ (from the base case of $125 \%$ ) to reflect Medicare-to-commercial payer ratios reported in the literature, ${ }^{17,18}$ while the charge-to-payment adjustment for hysterectomy and myomectomy arms was also decreased down to $50 \%$ (from the base case of $70 \%$ ), to assess the robustness of results. Payer costs for the hysterectomy and myomectomy arms were stratified by inpatient or outpatient setting, as well as by robotic-assisted status, to explore how these factors affect costs. Finally, sensitivity analyses were conducted in which the high-cost outliers, or those costs that were more than 1.5 times median, were removed from the hysterectomy and myomectomy arms.

\section{Analysis}

All analyses were conducted in Stata (version 13; StataCorp LLC, College Station, Texas). Patient characteristics were reported, and a chi-square analysis was used to determine the difference between groups. Means for total payments, payments for the index procedure and length of stay (LOS, defined as time of admission to time of discharge or time eligible to be discharged), and payments for postoperative HCRU were reported for the TFA, hysterectomy, and myomectomy procedures. The Welch $t$-test for unequal variances were used to compare mean facility costs between TFA and the comparators. For all comparisons, a p-value $<0.05$ was considered statistically significant.

\section{Results}

\section{Patient Characteristics and LOS}

In the TFA arm, 6 outpatient hospital sites from the SONATA trial participated in INSPIRE and provided billing data on 51 patients. For the comparator arms, a total of 35,463 patients who underwent hysterectomy and 8,548 patients who underwent myomectomy were included.

Table 2 describes the characteristics of patients included in the study. Women undergoing treatment with the Sonata system, with a mean age of 44 (CI: 43.0-45.0) years, were older than those undergoing either a hysterectomy (42; CI:

Table 2 Characteristics of Study Patients

\begin{tabular}{|c|c|c|c|c|c|c|c|}
\hline $\begin{array}{l}\text { Parameter Mean } \pm \\
\text { SD }\end{array}$ & $\begin{array}{l}\text { TFA } \\
n=51\end{array}$ & $95 \% \mathrm{Cl}$ & $\begin{array}{l}\text { Hysterectomy } \\
n=35,463\end{array}$ & $95 \% \mathrm{Cl}$ & $\begin{array}{l}\text { Myomectomy } \\
n=8,548\end{array}$ & $95 \% \mathrm{Cl}$ & $p$-value \\
\hline Age in years & $44 \pm 3.7$ & $43.0-45.0$ & $42 \pm 5.0$ & $41.9-42.1$ & $39 \pm 5.9$ & $38.9-39.1$ & $<0.001$ \\
\hline LOS in hours* & $5 \pm 1.7$ & $4.4-5.6$ & $73 \pm 39.1$ & $72.6-73.4$ & $79 \pm 34.6$ & $78.2-79.8$ & $<0.001$ \\
\hline \multicolumn{8}{|l|}{ Region n (\%) } \\
\hline Northeast & II (2I.6\%) & & 4,165 (1I.7\%) & & I,86| (2I.8\%) & & $<0.001$ \\
\hline North central & 22 (43.1\%) & & 6,992 (19.7\%) & & I,349 (I5.8\%) & & \\
\hline South & 9 (I7.7\%) & & 18,925 (53.4\%) & & 4,007 (46.9\%) & & \\
\hline West & 9 (I7.7\%) & & 5,134 (14.5\%) & & I,278 (I5.0\%) & & \\
\hline Unknown & - & & 247 (0.7\%) & & $53(0.6 \%)$ & & \\
\hline
\end{tabular}

Notes: $p$-values for age and LOS was calculated using $t$-test assuming unequal variances, and for region calculated using chi-square test. *LOS was only available for inpatient hysterectomy $(n=11,166)$ and myomectomy $(n=2,259)$ patients. All other measurements were captured for all patients.

Abbreviations: TFA, transcervical fibroid ablation; LOS, Length of stay.

Table 3 Postoperative Health Care Resource Utilization (HCRU)

\begin{tabular}{|c|c|c|c|c|c|}
\hline \multirow[t]{2}{*}{ HCRU Frequency (\%) } & TFA & Hysterectomy & \multirow[t]{2}{*}{$p$-value } & Myomectomy & \multirow[t]{2}{*}{$p$-value } \\
\hline & $n=51$ & $n=35,463$ & & $n=8,548$ & \\
\hline Subsequent Procedure ${ }^{\dagger}$ & I (2.0) & $2,201(6.2)$ & 0.209 & $493(5.8)$ & 0.244 \\
\hline \multicolumn{6}{|l|}{ Prescriptions } \\
\hline Opioids & $16(31.4)$ & I5,757 (44.4) & 0.061 & $3,212(37.6)$ & 0.362 \\
\hline NSAID & $2 I(4 I .2)$ & $12,752(36.0)$ & 0.438 & $2,650(31.0)$ & 0.117 \\
\hline Outpatient office visits (total) & $5(9.8)$ & $1,203(3.4)$ & 0.012 & $4,278(50.1)$ & $<0.001$ \\
\hline
\end{tabular}

Notes: $p$-value: test of proportions (z-test), each comparator versus Sonata. †Subsequent procedures (following the initial procedure) included myomectomy, hysterectomy, uterine artery embolization, MR-guided focused ultrasound, endometrial ablation, or trachelectomy.

Abbreviation: TFA, transcervical fibroid ablation. 
Table 4 Payments Through the I-Year Postoperative Period

\begin{tabular}{|c|c|c|c|c|c|c|c|c|}
\hline \multirow[t]{2}{*}{ Total Payments Mean (SD) } & \multirow{2}{*}{$\frac{\text { TFA }}{n=51}$} & \multirow[t]{2}{*}{$95 \% \mathrm{Cl}$} & \multirow{2}{*}{$\begin{array}{l}\text { Hysterectomy } \\
n=35,463\end{array}$} & \multirow[t]{2}{*}{$95 \% \mathrm{Cl}$} & \multirow[t]{2}{*}{$p$-value } & \multirow{2}{*}{$\begin{array}{l}\text { Myomectomy } \\
n=8,548\end{array}$} & \multirow[t]{2}{*}{$95 \% \mathrm{Cl}$} & \multirow[t]{2}{*}{ p-value } \\
\hline & & & & & & & & \\
\hline Total payments (\$) & $8,941(448.9)$ & $8,815-9,067$ & $24,156(16,695.3)$ & $23,982-24,330$ & $<0.001$ & $22,784(19,341.7)$ & $22,374-23,194$ & $<0.001$ \\
\hline Index procedure & 8,797 (184.8) & $8,745-8,849$ & $23,457(15,753.5)$ & $23,293-23,621$ & $<0.001$ & $21,340(18,499.0)$ & $20,948-21,732$ & $<0.001$ \\
\hline Postoperative & $143(427.1)$ & $23-263$ & $699(4,983.10)$ & $647-751$ & $<0.001$ & $\mathrm{I}, 444(5,283.5)$ & I,332-I,556 & $<0.001$ \\
\hline Complication-related & $0(0.0)$ & $0-0$ & $289(4,640.4)$ & $24 I-337$ & $<0.001$ & $14(506.3)$ & $3-25$ & 0.011 \\
\hline Office Visits ${ }^{\dagger}$ & $9(27.8)$ & $1-17$ & $4(66.5)$ & $3-5$ & 0.210 & $99(1,443.6)$ & $68-130$ & $<0.001$ \\
\hline Subsequent Procedure & $59(417.8)$ & $-59-177$ & $117(710.8)$ & $110-124$ & 0.224 & $135(868.6)$ & $117-153$ & 0.206 \\
\hline Medications & $75(207.3)$ & $17-133$ & $236(1,548.6)$ & $220-252$ & $<0.001$ & $881(4,453.4)$ & 787-975 & $<0.001$ \\
\hline Radiology & $6(46.2)$ & $-7-19$ & 52 (291.4) & $49-55$ & $<0.001$ & $314(935.3)$ & $294-334$ & $<0.001$ \\
\hline
\end{tabular}

Notes: $p$-value: $t$-test assuming unequal variances was used to determine statistical significance, each comparator versus Sonata. †Defined differently for TFA vs Hysterectomy and Myomectomy; TFA=office visits other than SONATA protocol defined visits; Hysterectomy/Myomectomy=office visit specific to UF.

Abbreviation: TFA, transcervical fibroid ablation.

41.9-42.1) or myomectomy (39; all $p<0.001$; CI: 38.9-39.1). Additionally, patients in the Sonata arm had a significantly shorter mean LOS for their procedure $(5 \pm 1.7 \mathrm{hrs}$; CI: 4.4-5.6), compared to those who underwent either hysterectomy or myomectomy ( $>3$ days; $p<0.001$; CI: $72.6-73.4$ and CI: 78.2-79.8).

\section{HCRU}

Table 3 shows the results from the 12-month postoperative HCRU review. HCRU categories included total outpatient office visits, subsequent procedure, and prescription medications (opioids and NSAIDs). As a determinant of pain control costing, $31.4 \%$ of TFA, $44.4 \%$ of hysterectomy, and $37.6 \%$ of myomectomy patients received an opioid prescription, although differences were not statistically significant. To gain further understanding of procedural outcomes and associated cost, total payments were broken down by index procedure and postoperative HCRU (Table 4).

Index procedure payments were significantly lower for TFA in comparison to both hysterectomy and myomectomy $(p<0.001)$. For total postoperative costs, Sonata

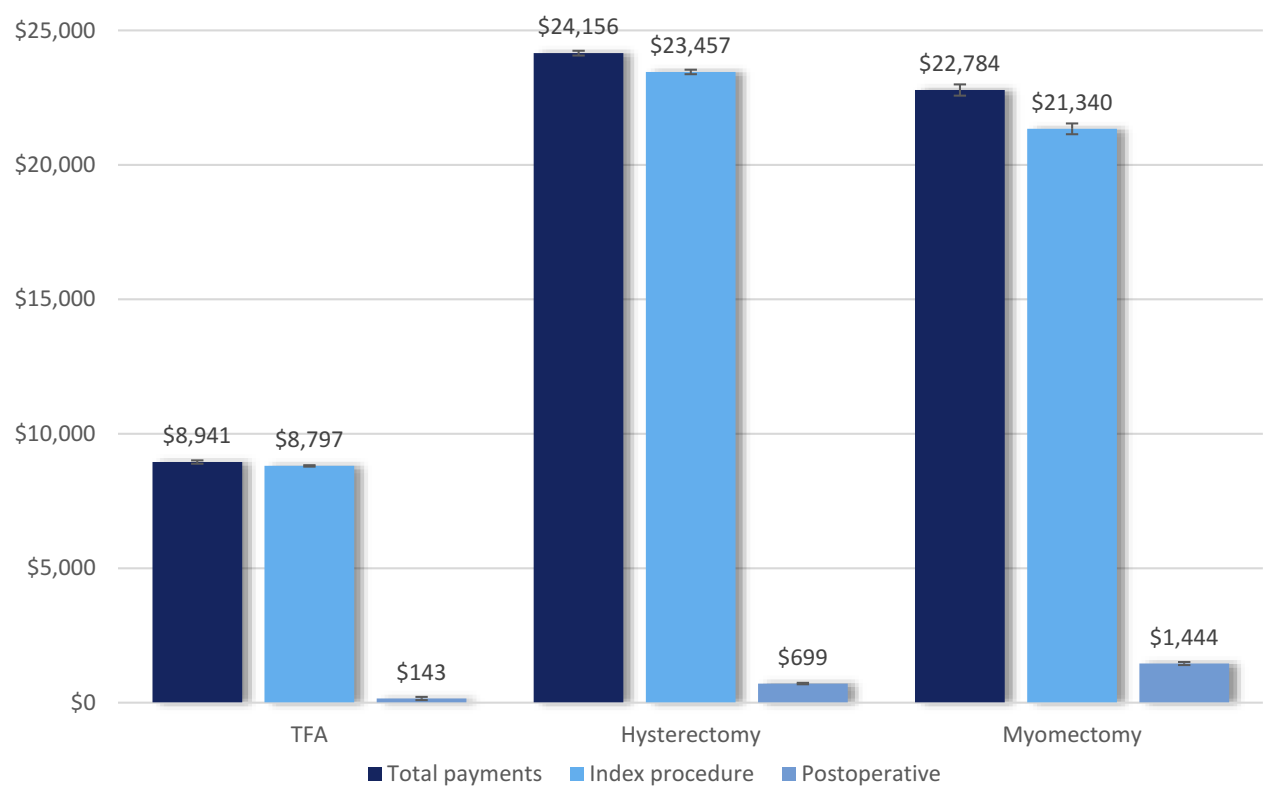

Figure I Mean payments per procedure ( \pm SE). Each payment category for Transcervical Fibroid Ablation was significantly less costly than the corresponding Hysterectomy or Myomectomy payment category.

Abbreviations: SE, standard error; TFA, transcervical fibroid ablation. 
patients, on average, had significantly lower total postoperative costs than both hysterectomy and myomectomy patients $(p<0.001)$. As shown in Table 4 , the mean costs of complications across all patients by study arm were $\$ 0$ (no complications) for TFA, \$289 for hysterectomy ( $p<0.001 ; \mathrm{CI}: \$ 241-\$ 337$ ), and \$14 for myomectomy patients $(p=0.011$; CI: $\$ 3-\$ 25)$. The mean cost associated with postoperative prescription use for TFA was \$75 (CI: \$17-\$133), which was significantly lower in comparison to both hysterectomy patients (\$236, $p<0.001$; CI: \$220-\$252) and myomectomy patients (\$881, $p<0.001 ;$ CI: \$787-\$975). TFA patients had mean outpatient office visit costs (\$9; CI: \$1-\$17) comparable to those of hysterectomy patients ( $\$ 4$, non- significant; CI: \$3-\$5), but significantly different from myomectomy patients (\$99, $p<0.001$; CI: $\$ 68-\$ 130)$. TFA patients had a mean cost of \$6 (CI: \$7-\$19) for radiology, with hysterectomy and myomectomy mean costs both significantly higher at $\$ 52(p<0.001$; CI: \$49-\$55) and \$314 $(p<0.001 ;$ CI: \$294-\$334), respectively.

\section{Payer Costs}

\section{Procedure Costs}

Results from the overall payer cost analysis are presented in Figure 1. The mean cost to the payer for TFA with Sonata and the associated 12-month postoperative HCRU was $\$ 8,941$ (CI: $\$ 8,815-\$ 9,067$ ), compared to $\$ 24,156$

Table 5 Breakdown of Hysterectomy and Myomectomy Payments by Robotic Assisted/Not Robotic Assisted and Inpatient/ Outpatient Status

\begin{tabular}{|c|c|c|c|c|c|c|c|}
\hline Technology & Setting & Robotic Assisted Status & Variable & Mean (\$) & SD (\$) & $95 \% \mathrm{Cl}$ & $p$-value \\
\hline TFA $\ddagger$ & Outpatient & Non-robotic assisted $(n=5 I)$ & $\begin{array}{l}\text { Total payments } \\
\text { Index procedure } \\
\text { Postoperative }\end{array}$ & $\begin{array}{l}8,941 \\
8,797 \\
143\end{array}$ & $\begin{array}{l}448.9 \\
184.8 \\
427.1\end{array}$ & $\begin{array}{l}8,815-9,067 \\
8,745-8,849 \\
23-263\end{array}$ & $\begin{array}{l}\text { Ref } \\
\text { Ref } \\
\text { Ref }\end{array}$ \\
\hline \multirow[t]{4}{*}{ Hysterectomy } & \multirow[t]{2}{*}{ Inpatient } & Robotic assisted $(n=\mid 42)$ & $\begin{array}{l}\text { Total payments } \\
\text { Index procedure } \\
\text { Postoperative }\end{array}$ & $\begin{array}{l}32,547 \\
31,864 \\
682\end{array}$ & $\begin{array}{l}19,166.5 \\
18,321.4 \\
4,490.4\end{array}$ & $\begin{array}{l}29,367-35,727 \\
28,825-34,903 \\
-62-1,428\end{array}$ & $\begin{array}{l}<0.001 \\
<0.001 \\
0.160\end{array}$ \\
\hline & & Non-robotic assisted $(n=\mid 1,024)$ & $\begin{array}{l}\text { Total payments } \\
\text { Index procedure } \\
\text { Postoperative }\end{array}$ & $\begin{array}{l}28,144 \\
27,161 \\
983\end{array}$ & $\begin{array}{l}19,421.0 \\
17,285.9 \\
8,078.8\end{array}$ & $\begin{array}{l}27,78 I-28,507 \\
26,838-27,484 \\
832-I, 134\end{array}$ & $\begin{array}{l}<0.001 \\
<0.001 \\
<0.001\end{array}$ \\
\hline & \multirow[t]{2}{*}{ Outpatient } & Robotic assisted $(n=2,362)$ & $\begin{array}{l}\text { Total payments } \\
\text { Index procedure } \\
\text { Postoperative }\end{array}$ & $\begin{array}{l}31,219 \\
30,633 \\
586\end{array}$ & $\begin{array}{l}19,674 . \mid \\
19,498.4 \\
1,728.4\end{array}$ & $\begin{array}{l}30,425-32,013 \\
29,846-31,420 \\
516-656\end{array}$ & $\begin{array}{l}<0.001 \\
<0.001 \\
<0.001\end{array}$ \\
\hline & & Non-robotic assisted $(n=21,935)$ & $\begin{array}{l}\text { Total payments } \\
\text { Index procedure } \\
\text { Postoperative }\end{array}$ & $\begin{array}{l}21,337 \\
20,769 \\
568\end{array}$ & $\begin{array}{l}\mid 3,975.0 \\
\mid 3,706.3 \\
2,6 \mid 4.5\end{array}$ & $\begin{array}{l}21,152-21,512 \\
20,588-20,950 \\
533-603\end{array}$ & $\begin{array}{l}<0.001 \\
<0.001 \\
<0.001\end{array}$ \\
\hline \multirow[t]{4}{*}{ Myomectomy } & \multirow[t]{2}{*}{ Inpatient } & Robotic assisted $(n=I \mid)$ & $\begin{array}{l}\text { Total payments } \\
\text { Index procedure } \\
\text { Postoperative }\end{array}$ & $\begin{array}{l}31,720 \\
30,966 \\
754\end{array}$ & $\begin{array}{l}\text { II,929.9 } \\
\text { II,737.3 } \\
1,056.4\end{array}$ & $\begin{array}{l}23,705-39,735 \\
23,04 I-38,89 I \\
45-I, 463\end{array}$ & $\begin{array}{l}<0.001 \\
<0.001 \\
0.087\end{array}$ \\
\hline & & Non-robotic assisted $(n=2,248)$ & $\begin{array}{l}\text { Total payments } \\
\text { Index procedure } \\
\text { Postoperative }\end{array}$ & $\begin{array}{l}30,814 \\
29,794 \\
1,020\end{array}$ & $\begin{array}{l}21,702.2 \\
21,416.7 \\
3,448.4\end{array}$ & $\begin{array}{l}29,916-31,712 \\
28,908-30,680 \\
877-1,163\end{array}$ & $\begin{array}{l}<0.001 \\
<0.001 \\
<0.001\end{array}$ \\
\hline & \multirow[t]{2}{*}{ Outpatient } & Robotic assisted $(n=287)$ & $\begin{array}{l}\text { Total payments } \\
\text { Index procedure } \\
\text { Postoperative }\end{array}$ & $\begin{array}{l}40,875 \\
38,810 \\
2,065\end{array}$ & $\begin{array}{l}29,556.7 \\
28,056.8 \\
7,614.7\end{array}$ & $\begin{array}{l}37,44 I-44,309 \\
35,550-42,070 \\
I, I 80-2,950\end{array}$ & $\begin{array}{l}<0.001 \\
<0.001 \\
<0.001\end{array}$ \\
\hline & & Non-robotic assisted $(n=6,002)$ & $\begin{array}{l}\text { Total payments } \\
\text { Index procedure } \\
\text { Postoperative }\end{array}$ & $\begin{array}{l}18,896 \\
17,32 \mid \\
I, 574\end{array}$ & $\begin{array}{l}16,109.5 \\
14,839.7 \\
5,695.4\end{array}$ & $\begin{array}{l}18,488-19,304 \\
16,945-17,697 \\
1,430-1,7 \mid 8\end{array}$ & $\begin{array}{l}<0.001 \\
<0.001 \\
<0.001\end{array}$ \\
\hline
\end{tabular}

Notes: $\mathrm{p}$-value: $t$-test assuming unequal variances was used to determine statistical significance. $\ddagger$ TFA with Sonata does not utilize robotic assistance, laparoscopic, or hysteroscopic guidance.

Abbreviation: TFA, transcervical fibroid ablation. 
Table 6 Sensitivity Analysis

\begin{tabular}{|c|c|c|c|c|c|c|}
\hline $\begin{array}{l}\text { Charge to Payment } \\
\text { Ratio }\end{array}$ & Technology & Variable & $\begin{array}{l}\text { Mean } \\
(\$)\end{array}$ & SD (\$) & $95 \% \mathrm{Cl}(\$)$ & p-value \\
\hline Site-specific & $\begin{array}{l}\text { TFA (I25\% Medicare to commercial } \\
\text { ratio) }\end{array}$ & $\begin{array}{l}\text { Total payments } \\
\text { Index procedure } \\
\text { Postoperative }\end{array}$ & $\begin{array}{l}8,94 \mid \\
8,797 \\
143\end{array}$ & $\begin{array}{l}448.9 \\
184.8 \\
427.1\end{array}$ & $\begin{array}{l}8,815-9,067 \\
8,745-8,849 \\
23-263\end{array}$ & $\begin{array}{l}\text { Ref } \\
\text { Ref } \\
\text { Ref }\end{array}$ \\
\hline \multirow[t]{2}{*}{$70 \%$ Base case } & Hysterectomy & $\begin{array}{l}\text { Total payments } \\
\text { Index procedure } \\
\text { Postoperative }\end{array}$ & $\begin{array}{l}24,156 \\
23,457 \\
699\end{array}$ & $\begin{array}{l}16,695.3 \\
15,753.5 \\
4,983.1\end{array}$ & $\begin{array}{l}24,154-24,158 \\
23,293-23,621 \\
647-75 \mid\end{array}$ & $\begin{array}{l}<0.001 \\
<0.001 \\
<0.001\end{array}$ \\
\hline & Myomectomy & $\begin{array}{l}\text { Total payments } \\
\text { Index procedure } \\
\text { Postoperative }\end{array}$ & $\begin{array}{l}22,784 \\
21,340 \\
I, 444\end{array}$ & $\begin{array}{l}19,341.7 \\
18,499.0 \\
5,283.5\end{array}$ & $\begin{array}{l}22,374-23,194 \\
20,948-21,732 \\
1,332-1,556\end{array}$ & $\begin{array}{l}<0.001 \\
<0.001 \\
<0.001\end{array}$ \\
\hline \multirow[t]{2}{*}{$60 \%$} & Hysterectomy & $\begin{array}{l}\text { Total payments } \\
\text { Index procedure } \\
\text { Postoperative }\end{array}$ & $\begin{array}{l}20,705 \\
20,106 \\
599\end{array}$ & $\begin{array}{l}|4,3| 0.3 \\
\mid 3,503.0 \\
4,27 \mid .2\end{array}$ & $\begin{array}{l}20,556-20,854 \\
19,965-20,247 \\
555-643\end{array}$ & $\begin{array}{l}<0.001 \\
<0.001 \\
<0.001\end{array}$ \\
\hline & Myomectomy & $\begin{array}{l}\text { Total payments } \\
\text { Index procedure } \\
\text { Postoperative }\end{array}$ & $\begin{array}{l}19,529 \\
18,29 \mid \\
1,238\end{array}$ & $\begin{array}{l}16,578.6 \\
15,856.3 \\
4,528.7\end{array}$ & $\begin{array}{l}|9,| 77-19,88 \mid \\
\mid 7,955-18,627 \\
I, \mid 42-1,334\end{array}$ & $\begin{array}{l}<0.001 \\
<0.001 \\
<0.001\end{array}$ \\
\hline \multirow[t]{2}{*}{$50 \%$} & Hysterectomy & $\begin{array}{l}\text { Total payments } \\
\text { Index procedure } \\
\text { Postoperative }\end{array}$ & $\begin{array}{l}17,254 \\
16,755 \\
499\end{array}$ & $\begin{array}{l}11,925.2 \\
11,252.5 \\
3,559.3\end{array}$ & $\begin{array}{l}17,130-17,378 \\
16,638-16,872 \\
462-536\end{array}$ & $\begin{array}{l}<0.001 \\
<0.001 \\
<0.001\end{array}$ \\
\hline & Myomectomy & $\begin{array}{l}\text { Total payments } \\
\text { Index procedure } \\
\text { Postoperative }\end{array}$ & $\begin{array}{l}16,274 \\
15,243 \\
I, 03 \mid\end{array}$ & $\begin{array}{l}13,8 \mid 5.5 \\
13,2 \mid 3.6 \\
3,773.9\end{array}$ & $\begin{array}{l}|5,98|-16,567 \\
\mid 4,963-15,523 \\
95|-|,|| \mid\end{array}$ & $\begin{array}{l}<0.001 \\
<0.001 \\
<0.001\end{array}$ \\
\hline Site-specific & $\begin{array}{l}\text { TFA ( } 140 \% \text { Medicare to commercial } \\
\text { ratio) }\end{array}$ & $\begin{array}{l}\text { Total payments } \\
\text { Index procedure } \\
\text { Postoperative }\end{array}$ & $\begin{array}{l}10,014 \\
9,853 \\
160\end{array}$ & $\begin{array}{l}502.8 \\
207.0 \\
478.4\end{array}$ & $\begin{array}{l}9,873-10,155 \\
9,795-9,911 \\
26-294\end{array}$ & $\begin{array}{l}\text { Ref } \\
\text { Ref } \\
\text { Ref }\end{array}$ \\
\hline \multirow[t]{2}{*}{$70 \%$ Base case } & Hysterectomy & $\begin{array}{l}\text { Total payments } \\
\text { Index procedure } \\
\text { Postoperative }\end{array}$ & $\begin{array}{l}24,156 \\
23,457 \\
699\end{array}$ & $\begin{array}{l}16,695.3 \\
15,753.5 \\
4,983.1\end{array}$ & $\begin{array}{l}24,154-24,158 \\
23,293-23,621 \\
647-751\end{array}$ & $\begin{array}{l}<0.001 \\
<0.001 \\
<0.001\end{array}$ \\
\hline & Myomectomy & $\begin{array}{l}\text { Total payments } \\
\text { Index procedure } \\
\text { Postoperative }\end{array}$ & $\begin{array}{l}22,784 \\
21,340 \\
I, 444\end{array}$ & $\begin{array}{l}19,341.7 \\
18,499.0 \\
5,283.5\end{array}$ & $\begin{array}{l}22,374-23,194 \\
20,948-21,732 \\
1,332-1,556\end{array}$ & $\begin{array}{l}<0.001 \\
<0.001 \\
<0.001\end{array}$ \\
\hline \multirow[t]{2}{*}{$60 \%$} & Hysterectomy & $\begin{array}{l}\text { Total payments } \\
\text { Index procedure } \\
\text { Postoperative }\end{array}$ & $\begin{array}{l}20,705 \\
20,106 \\
599\end{array}$ & $\begin{array}{l}|4,3| 0.3 \\
\mid 3,503.0 \\
4,27 \mid .2\end{array}$ & $\begin{array}{l}20,556-20,854 \\
19,965-20,247 \\
555-643\end{array}$ & $\begin{array}{l}<0.001 \\
<0.001 \\
<0.001\end{array}$ \\
\hline & Myomectomy & $\begin{array}{l}\text { Total payments } \\
\text { Index procedure } \\
\text { Postoperative }\end{array}$ & $\begin{array}{l}19,529 \\
18,29 \mid \\
1,238\end{array}$ & $\begin{array}{l}16,578.6 \\
15,856.3 \\
4,528.7\end{array}$ & $\begin{array}{l}19,|77-19,88| \\
\mid 7,955-18,627 \\
1, \mid 42-1,334\end{array}$ & $\begin{array}{l}<0.001 \\
<0.001 \\
<0.001\end{array}$ \\
\hline \multirow[t]{2}{*}{$50 \%$} & Hysterectomy & $\begin{array}{l}\text { Total payments } \\
\text { Index procedure } \\
\text { Postoperative }\end{array}$ & $\begin{array}{l}17,254 \\
16,755 \\
499\end{array}$ & $\begin{array}{l}11,925.2 \\
11,252.5 \\
3,559.3\end{array}$ & $\begin{array}{l}17,130-17,378 \\
16,638-16,872 \\
462-536\end{array}$ & $\begin{array}{l}<0.001 \\
<0.001 \\
<0.001\end{array}$ \\
\hline & Myomectomy & $\begin{array}{l}\text { Total payments } \\
\text { Index procedure } \\
\text { Postoperative }\end{array}$ & $\begin{array}{l}16,274 \\
15,243 \\
I, 03 \mid\end{array}$ & $\begin{array}{l}13,815.5 \\
13,213.6 \\
3,773.9\end{array}$ & $\begin{array}{l}|5,98|-\mid 6,567 \\
\mid 4,963-15,523 \\
95|-|,|| \mid\end{array}$ & $\begin{array}{l}<0.001 \\
<0.001 \\
<0.001\end{array}$ \\
\hline
\end{tabular}

(Continued) 
Table 6 (Continued).

\begin{tabular}{|c|c|c|c|c|c|c|}
\hline $\begin{array}{l}\text { Charge to Payment } \\
\text { Ratio }\end{array}$ & Technology & Variable & $\begin{array}{l}\text { Mean } \\
(\$)\end{array}$ & SD (\$) & $95 \% \mathrm{Cl}(\$)$ & p-value \\
\hline Site-specific & $\begin{array}{l}\text { TFA ( } 188 \% \text { Medicare to commercial } \\
\text { ratio) }\end{array}$ & $\begin{array}{l}\text { Total payments } \\
\text { Index procedure } \\
\text { Postoperative }\end{array}$ & $\begin{array}{l}\mid 3,447 \\
|3,23| \\
215\end{array}$ & $\begin{array}{l}675.2 \\
278.0 \\
642.4\end{array}$ & $\begin{array}{l}13,257-13,637 \\
13,153-13,309 \\
34-396\end{array}$ & $\begin{array}{l}\text { Ref } \\
\text { Ref } \\
\text { Ref }\end{array}$ \\
\hline \multirow[t]{2}{*}{$70 \%$ Base case } & Hysterectomy & $\begin{array}{l}\text { Total payments } \\
\text { Index procedure } \\
\text { Postoperative }\end{array}$ & $\begin{array}{l}24,156 \\
23,457 \\
699\end{array}$ & $\begin{array}{l}16,695.3 \\
15,753.5 \\
4,983.1\end{array}$ & $\begin{array}{l}24,154-24,158 \\
23,293-23,62 \mid \\
647-75 \mid\end{array}$ & $\begin{array}{l}<0.001 \\
<0.001 \\
<0.001\end{array}$ \\
\hline & Myomectomy & $\begin{array}{l}\text { Total payments } \\
\text { Index procedure } \\
\text { Postoperative }\end{array}$ & $\begin{array}{l}22,784 \\
21,340 \\
1,444\end{array}$ & $\begin{array}{l}19,341.7 \\
18,499.0 \\
5,283.5\end{array}$ & $\begin{array}{l}22,374-23,194 \\
20,948-21,732 \\
1,332-1,556\end{array}$ & $\begin{array}{l}<0.001 \\
<0.001 \\
<0.001\end{array}$ \\
\hline \multirow[t]{2}{*}{$60 \%$} & Hysterectomy & $\begin{array}{l}\text { Total payments } \\
\text { Index procedure } \\
\text { Postoperative }\end{array}$ & $\begin{array}{l}20,705 \\
20,106 \\
599\end{array}$ & $\begin{array}{l}|4,3| 0.3 \\
\mid 3,503.0 \\
4,27 \mid .2\end{array}$ & $\begin{array}{l}20,556-20,854 \\
19,965-20,247 \\
555-643\end{array}$ & $\begin{array}{l}<0.001 \\
<0.001 \\
<0.001\end{array}$ \\
\hline & Myomectomy & $\begin{array}{l}\text { Total payments } \\
\text { Index procedure } \\
\text { Postoperative }\end{array}$ & $\begin{array}{l}19,529 \\
18,291 \\
1,238\end{array}$ & $\begin{array}{l}16,578.6 \\
15,856.3 \\
4,528.7\end{array}$ & $\begin{array}{l}19,177-19,881 \\
17,955-18,627 \\
1,142-1,334\end{array}$ & $\begin{array}{l}<0.001 \\
<0.001 \\
<0.001\end{array}$ \\
\hline \multirow[t]{2}{*}{$50 \%$} & Hysterectomy & $\begin{array}{l}\text { Total payments } \\
\text { Index procedure } \\
\text { Postoperative }\end{array}$ & $\begin{array}{l}17,254 \\
16,755 \\
499\end{array}$ & $\begin{array}{l}11,925.2 \\
11,252.5 \\
3,559.3\end{array}$ & $\begin{array}{l}17,130-17,378 \\
16,638-16,872 \\
462-536\end{array}$ & $\begin{array}{l}<0.001 \\
<0.001 \\
0.003\end{array}$ \\
\hline & Myomectomy & $\begin{array}{l}\text { Total payments } \\
\text { Index procedure } \\
\text { Postoperative }\end{array}$ & $\begin{array}{l}16,274 \\
\mid 5,243 \\
|, 03|\end{array}$ & $\begin{array}{l}13,8 \mid 5.5 \\
13,2 \mid 3.6 \\
3,773.9\end{array}$ & $\begin{array}{l}|5,98|-\mid 6,567 \\
\mid 4,963-15,523 \\
951-1,1||\end{array}$ & $\begin{array}{l}<0.001 \\
<0.001 \\
<0.001\end{array}$ \\
\hline
\end{tabular}

Notes: $p$-value: $t$-test assuming unequal variances was used to determine statistical significance, each comparator versus Sonata.

Abbreviation: TFA, transcervical fibroid ablation.

(CI: \$23,982-\$24,330) for hysterectomy and \$22,784 (CI: $\$ 22,374-\$ 23,194)$ for myomectomy. When compared to TFA, both hysterectomy and myomectomy incurred significantly higher payer costs $(p<0.001)$.

\section{Payment Sub-Analyses}

Table 5 presents payer costs stratified by both the use of robotic-assistance and the site of service (inpatient or outpatient). The least-costly hysterectomy procedures are those conducted in the outpatient setting without robotic assistance (mean cost of \$21,337; CI: \$21,152-\$21,512), while the least costly myomectomy procedures are also conducted in the outpatient setting, again without robotic assistance (mean cost of $\$ 18,896$; CI: $\$ 18,488-\$ 19,304)$. However, TFA continued to have significantly lower payer costs compared to these least costly comparator procedures $(p<0.001)$.

\section{Sensitivity Analyses}

A variety of one-way and two-way sensitivity analyses altered the Medicare-to-commercial payment ratios (ranging from the base case of $125 \%$ up to $188 \%$ ) and charge-to payment ratios (ranging from the base case of $70 \%$ down to $50 \%$ ) to explore how market changes affect payer costs of uterine fibroid treatment (Table 6). The mean payer cost for TFA rose to $\$ 13,447$ (CI: $\$ 13,257-\$ 13,637$ ) when the Medicare-to-commercial payment ratio increased to $188 \%$. Mean payer costs for hysterectomy and myomectomy fell to \$17,254 (CI: \$17,130-\$17,378) and \$16,274 (CI: \$15,981$\$ 16,567)$, respectively, when the charge-to-payment ratio for the comparator arms is reduced from $70 \%$ to $50 \%$. However, the mean total cost difference between TFA and both hysterectomy and myomectomy remained statistically significant in each of these analyses $(p<0.001)$, demonstrating significantly lower costs associated with TFA.

\section{Consideration of High Cost Outliers}

Figure 2 presents payer costs when high-cost outliers are removed from the hysterectomy and myomectomy cohorts. High-cost outliers for comparator arms were identified as procedures with total costs more than 1.5 times the median, resulting in thresholds of $\$ 48,541$ and $\$ 51,840$ for 
hysterectomy and myomectomy, respectively. With highcost outliers removed, the mean total payments associated with hysterectomy decreased to $\$ 21,268$ (CI: $\$ 21,152$ $\$ 21,384)$ and the mean total payments associated with myomectomy decreased to $\$ 19,388$ (CI: $\$ 19,129-\$ 19,647$ ). With high-cost outliers removed from the comparator arms, TFA continued to maintain significantly lower mean total payer costs $(\$ 8,941, p<0.001 ; \mathrm{CI}: \$ 8,815-\$ 9,067)$.

\section{Discussion}

The results of the INSPIRE study, which examined the health economic outcomes and costs of TFA using the Sonata system compared to hysterectomy and myomectomy, showed that the cost of TFA to payers is significantly less than that of hysterectomy and myomectomy. Based on the current analysis, the adoption of the TFA procedure may lead to a $60 \%$ reduction in payer costs when compared to the current prevalent surgical treatments for uterine fibroids. In an environment of increasing health care costs, it is important to understand the outcomes and costs associated with innovative new treatment options relative to the current standard(s) of care. While the safety and effectiveness of the Sonata procedure has been verified, ${ }^{13}$ there have yet to be published data comparing health economic outcomes, medical resource utilization, and payer costs of the procedure to the current standard treatments of hysterectomy and myomectomy.

This is the first study to examine 12-month payer costs and HCRU for TFA using the Sonata system and compared to those for hysterectomy and myomectomy for treatment of uterine fibroids. One important strength of the study is that a well-defined methodology for estimating payments was utilized, with extensive sensitivity analyses performed around estimation parameters. Additionally, the hysterectomy and myomectomy populations are well represented in the study, as evidenced by the large sample sizes in these arms.

Despite these strengths, some notable limitations must be acknowledged. Because Sonata payment data were obtained from a subset of sites that participated in the clinical trial, the sample size was small. Also, as described previously, several assumptions had to be made about payments. However, it should be noted that hysterectomy and myomectomy payer costs identified in this study were consistent with other studies in the literature. Research published by O'Sullivan et al found that hysterectomy results in mean payer-perspective costs of $\$ 19,800$ (in 2005 dollars); ${ }^{19}$ this is equivalent to $\$ 26,266$ in 2016 dollars and similar to the results noted here. ${ }^{20}$ Other US

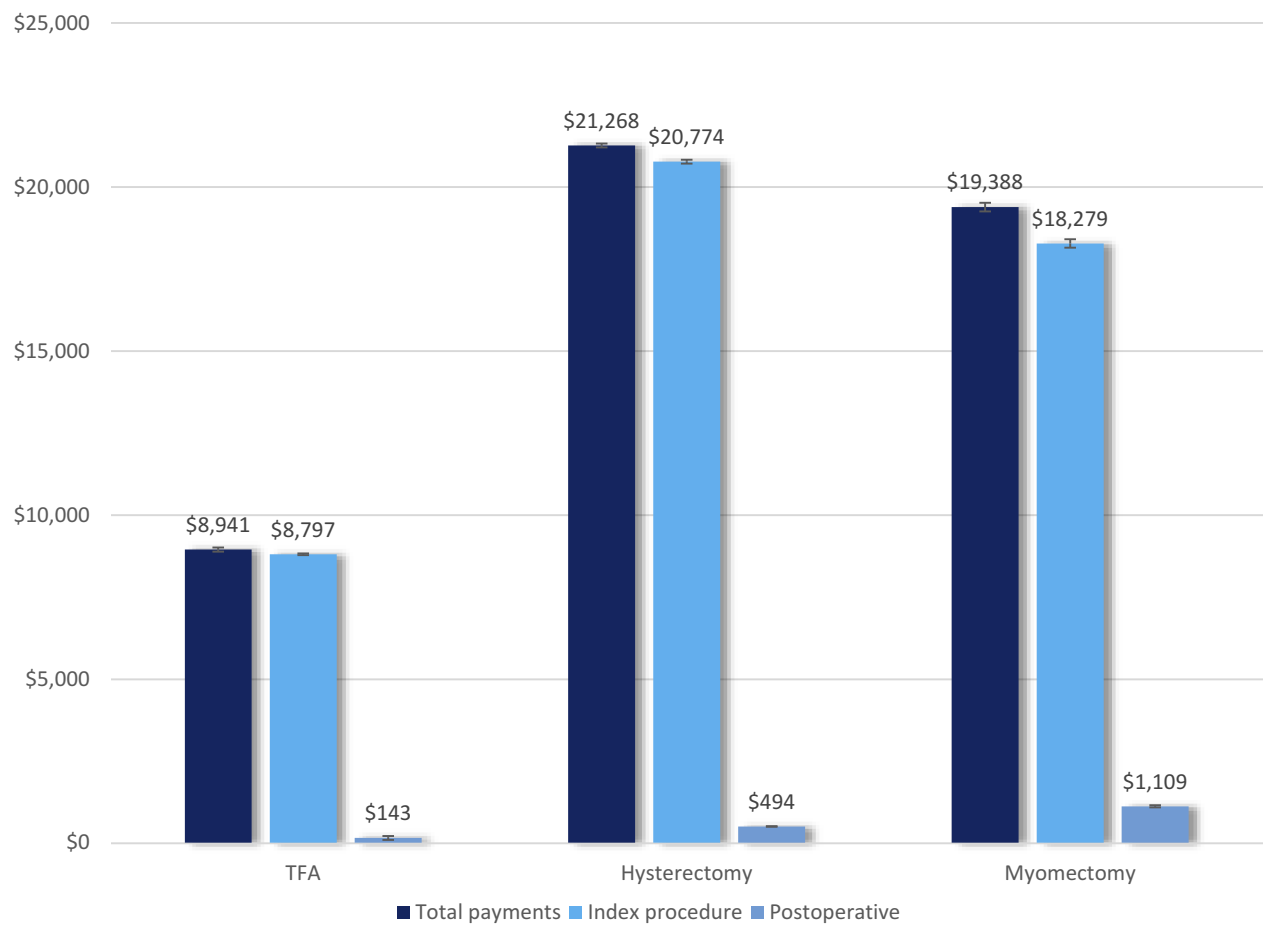

Figure 2 Mean payments per procedure with high cost outliers excluded from hysterectomy and myomectomy arms ( \pm SE). Each payment category for Transcervical Fibroid Ablation was significantly less costly than the corresponding Hysterectomy or Myomectomy payment category. Hysterectomy: Total payments> \$48,54I.2I excluded. Myomectomy: Total payments $>\$ 51,839.99$ excluded.

Abbreviations: SE, standard error; TFA, transcervical fibroid ablation. 
studies from 2000 and 2013 reviewed by Soliman produced similar 12-month payer costs, ranging from $\$ 19,000-\$ 20,000$ (\$20,588-\$21,649 in 2016 dollars) for hysterectomy and \$17,500-\$19,300 (\$18,996-\$20,907 in 2016 dollars) for myomectomy. ${ }^{21}$

Fitch and colleagues found that hysterectomy costs ranged from $\$ 13,000$ to $\$ 18,000$, which are lower than we estimated for hysterectomy. However, their analyses only looked at costs up to 30 days, while we examined costs through 12 -months. ${ }^{8}$ They also capped readmission costs at $\$ 100,000$. Our costs were closer to Bonafede et al, who found one-year hysterectomy costs to be up to $\$ 24,000 .^{9}$ Bonafede also looked at hysteroscopic myomectomy, which carried a mean cost of $\$ 17,300$, considerably higher than the cost associated with TFA $(\$ 8,914){ }^{9}$

\section{Conclusion}

Compared to hysterectomy and myomectomy, TFA treatment with the Sonata system was associated with significantly lower index procedure cost, complication cost, and LOS, contributing to a significantly lower total payer cost through 12 months.

\section{Funding}

Funding and support were provided by Gynesonics, Inc.

\section{Disclosure}

Dr Elizabeth Brooks, Dr Matt Yuen, Dr Robert L Bauserman, Miss Carter A Little, and Dr April ZambelliWeiner report their institution, TTi Health Research \& Economics, received research grants from Gynesonics Inc. during the conduct of the study. Dr Linda Mihalov reports personal fees from Gynesonics Inc., outside of the submitted work. The authors report no other conflicts of interest in this work.

\section{References}

1. Williams VSL, Jones G, Mauskopf J, Spalding J, Duchane J. Uterine fibroids: a review of health-related quality of life assessment. $J$ Womens Health. 2006;15(7):818-829. doi:10.1089/jwh.2006.15.818

2. Baird DD, Dunson DB, Hill MC, Cousins D, Schectman JM. High cumulative incidence of uterine leiomyoma in black and white women: ultrasound evidence. Am J Obstet Gynecol. 2003;188(1):100-107. doi: $10.1067 / \mathrm{mob} .2003 .99$

3. Lagana AS, Vergara D, Favilli A, et al. Epigenetic and genetic landscape of uterine leiomyomas: a current view over a common gynecological disease. Arch Gynecol Obstet. 2017;296:855-867. doi:10.1007/ s00404-017-4515-5
4. Barrett ML, Weiss AJ, Stocks C, Steiner CA, Myers ER. Procedures to treat Benign Uterine Fibroids in hospital inpatient and hospitalbased ambulatory surgery settings, 2013: statistical brief \#200. In: Healthcare Cost and Utilization Project (HCUP) Statistical Briefs. Rockville (MD): Agency for Healthcare Research and Quality (US); 2006. Available from: http://www.ncbi.nlm.nih.gov/books/ NBK349622/. Accessed February 2, 2018.

5. Brummer T, Jalkanen J, Fraser J, et al. FINHYST, a prospective study of 5279 hysterectomies: complications and their risk factors. Hum Reprod. 2011;26(7):1741-1751. doi:10.1093/humrep/der116

6. Prapas Y, Kalogiannidis I, Prapas N. Laparoscopy vs laparoscopically assisted myomectomy in the management of uterine myomas: aprospective study. Am J Obstet Gynecol. 2009;200(2):144.e1-144.e6. doi:10.1016/j.ajog.2008.08.063

7. Kong CY, Omer ZB, Pandharipande PV, et al. MRI-guided focused ultrasound surgery for uterine fibroid treatment: a cost-effectiveness analysis. AJR Am J Roentgenol. 2014;203(2):361-371. doi:10.2214/ AJR.13.11446

8. Fitch K, Huh W, Bochner A. Open vs. minimally invasive hysterectomy: commercially insured costs and readmissions. Manag Care Langhorne Pa. 2016;25(8):40-47.

9. Bonafede MM, Pohlman SK, Miller JD, Thiel E, Troeger KA, Miller CE. Women with newly diagnosed uterine fibroids: treatment patterns and cost comparison for select treatment options. Popul Health Manag. 2018;21(S1):S13-S20. doi:10.1089/pop.2017.0151

10. Cain-Nielsen AH, Moriarty JP, Stewart EA, Borah BJ. Costeffectiveness of uterine-preserving procedures for the treatment of uterine fibroid symptoms in the USA. J Comp Eff Res. 2014;3 (5):503-514. doi:10.2217/cer.14.32

11. Desormeaux P Gynecological Devices. United States: Decision Resources Group; 2018. Available from: https://decisionresour cesgroup.com/report/576432-medtech-gynecological-devicesmedtech-360-market/.Accessed December 11, 2019.

12. Lagana AS, Pacheco LA, Tinelli A, et al. Management of asymptomatic submucous myomas in women of reproductive age. A consensus statement from the global congress on hysteroscopy scientific committee. J Minim Invasive Gynecol. 2019;26(3):381-383. doi:10.1016/j.jmig. 2018.06.020

13. Chudnoff S, Guido R, Roy K, Levine D, Mihalov L, Garza-Leal J. Ultrasound-guided transcervical ablation of uterine leiomyomas. Obstet Gynecol. 2019;133(1):13-22. doi:10.1097/AOG.00000000 00003032

14. Medicare and commercial health insurance: the fundamental difference. Available from: https://www.healthaffairs.org/do/10. 1377/hblog20120215.016980/full/. Accessed July 25, 2018.

15. Truven health analytics.

16. Smith MW, Friedman B, Karaca Z, Wong HS. Predicting inpatient hospital payments in the United States: a retrospective analysis. $B M C$ Health Serv Res. 2015;15:372. doi:10.1186/s12913-015-1040-8

17. Clemens J, Gottlieb JD. In the shadow of a giant: medicare's influence on private physician payments. J Polit Econ. 2016;125(1):1-39. doi:10.1086/689772

18. Maeda J, Nelson L An analysis of private-sector prices for hospital admissions. Working Paper 2017-02. April 2017.

19. O'Sullivan A, Thompson D, Chu P, Lee DW, Stewart EA, Weinstein MC. Cost-effectiveness of magnetic resonance guided focused ultrasound for the treatment of uterine fibroids. Int J Technol Assess Health Care. 2009;25(1):14-25. doi:10.1017/S02664623090 90035

20. CPI inflation calculator. Available from: https://www.bls.gov/data/ inflation_calculator.htm. Accessed February 2, 2018.

21. Soliman AM, Yang H, Du EX, Kelkar SS, Winkel C. The direct and indirect costs of uterine fibroid tumors: a systematic review of the literature between 2000 and 2013. Am J Obstet Gynecol. 2015;213 (2):141-160. doi:10.1016/j.ajog.2015.03.019 


\section{Publish your work in this journal}

ClinicoEconomics and Outcomes Research is an international, peerreviewed open-access journal focusing on Health Technology Assessment, Pharmacoeconomics and Outcomes Research in the areas of diagnosis, medical devices, and clinical, surgical and pharmacological intervention. The economic impact of health policy and health systems organization also constitute important areas of coverage. The manuscript management system is completely online and includes a very quick and fair peer-review system, which is all easy to use. Visit $\mathrm{http}: / /$ www.dovepress.com/testimonials.php to read real quotes from published authors. 